\title{
Chromosome 17 centromere amplification and chromosomal instability (CIN) in breast cancer: Pathogenic and therapeutic implications
}

\author{
Minireview
}

I. A. VOUTSADAKIS ${ }^{1,2, *}$

Algoma District Cancer Program, Sault Area Hospital, Sault Ste. Marie, Ontario, Canada; Section of Internal Medicine, Division of Clinical Sciences, Northern Ontario School of Medicine, Sudbury, Ontario, Canada

*Correspondence: ivoutsadakis@yahoo.com;ivoutsadakis@nosm.ca

Received March 9, 2019 / Accepted May 22, 2019

\begin{abstract}
Chromosomal instability (CIN) is present in variable degrees in a significant percentage (up to 90\%) of cancers and often portends adverse outcomes. However, it has not been incorporated in clinical practice as a prognostic marker due to the lack of standardization and proof of clinical utility of assays to measure it, as well as uncertainties regarding optimal cut-offs. Amplification of the centromeric region of chromosome 17 as measured by In Situ Hybridization (ISH) of the CEP17 probe is used clinically as part of the ISH assay for HER2 status determination in breast cancer in cases with intermediate (2+) result of HER2 protein expression by immunohistochemistry. CEP17 amplification concerns the centromeric area and rarely extends beyond it to involve polysomy of the whole chromosome. The association of CEP17 amplification with generalized CIN remains uncertain. Such association, if confirmed, could be an opportunity for a practical and clinically validated test of CIN in breast cancer. This paper explores the association of CIN with centromere 17 amplification and with centromere function in general, as well as the pathophysiology of centromeres/kinetochore function during mitosis that underlies their relationship with CIN in cancer and in breast cancer in particular.
\end{abstract}

Key words: chromosomal instability, aneuploidy, breast cancer, centrosome 17, prognosis

Chromosomal instability (CIN) is defined as a gain or a loss of chromosomal material in one or more chromosomes. Gain or loss of whole chromosomes is referred to as aneuploidy or numerical CIN (polysomy or monosomy). Amplifications or deletions containing only parts of chromosomes are referred to as structural CIN [1]. Most commonly, numeric aneuploidy is associated with structural aneuploidy through a production of micronuclei that contain lagging chromosomes produced through mitotic defects. These micronuclei may subsequently break down and release their fragmented DNA content that can become re-incorporated in chromosomes of the main nucleus [2]. CIN is common in cancers and its incidence in breast cancer is high. In a study, for example using centromere 2 and 15 probes, $36.9 \%$ of cases (82 of 222 patients) had deviations from the modal number in these centromeres in $30 \%$ or more of cells [3]. In another study, $62.3 \%$ of 313 breast cancer samples showed gains in either chromosome 1 or chromosome 8 , or both [4].
The common amplification of ERBB2 locus in breast cancers, that occurs in about $15 \%$ of these cancers and defines a clinical sub-type, is an example of a structural aneuploidy. In these cancers a genomic area surrounding the ERBB2 locus, termed ERBB2 amplicon, is amplified without amplification of loci in chromosome 17 beyond the amplicon $[5,6]$. The size of the ERBB2 amplicon is variable. It has been observed that, as expected, genes localized closer to the ERBB2 locus are most commonly co-amplified compared with more distantly located genes at 17q12-21 loci [7, 8]. Similarly, cases that show amplifications of the centromeric area of chromosome 17, as identified with the CEP17 probe (Centromere Enumeration Probe 17), concern almost always amplification of this area only, and are very rarely associated with polysomy 17 [9-11]. According to the ASCO-CAP (American Society of Clinical Oncology and College of American Pathologists) definition of ERBB2 amplification for clinical use, if there exists a more widespread amplifica- 
tion or co-amplification that includes the centromeric area of chromosome 17 (CEP17) and thus producing an ERBB2 to CEP17 ratio in dual probe ISH evaluation of less than 2, ERBB2 is considered non-amplified except if its absolute copy number exceeds 6 [12]. This definition attempts to capture the fact that cancers with widespread amplifications may derive reduced or no benefit from HER2-targeting treatments, despite increased ERBB2 copy numbers. The possible different pathogenic implications of more extensive amplifications contributing to this resistance remain ill-defined. Reduced benefit of HER2-targeting treatments in patients that present with concomitant increase of copy numbers of CEP17 region may imply different pathogenesis that makes these cancers less dependent on function of the amplified HER2 protein and thus less sensitive to its inhibition. Alternatively, increase of copy numbers of CEP17 region may denote an underlying higher degree of generalized CIN that results in HER2-targeting treatments resistance. This is despite the fact that even ERBB2 amplified, as clinically defined, breast cancers contain an increased number of concomitant chromosomal gains compared to non-amplified counterparts [13]. This paper will discuss the role of centromere 17 alterations in breast cancer both regarding pathogenesis and therapeutic implications. Associations of centromere 17 alterations with CIN will also be discussed.

\section{Centromere 17 amplification in breast cancer: Associations and prognostic implications}

Amplification of the centromeric region of chromosome 17 is measured in clinical samples of breast cancer by ISH using the probe D17Z1 that hybridizes with a satellite DNA repeats present in tandem arrays across human chromosome 17 centromere. D17Z1 arrays are the site of kinetochore assembly of human chromosome 17 during mitosis in most cases, although a back-up array assembles the kinetochore in a minority of cases [14]. Active centromeric regions that assemble kinetochores during mitosis play a critical role in correct spindle construction and equal chromosome distribution in daughter cells [15]. Thus, cancers with CEP17 numeric abnormalities may have a parallel general- ized defect in the mitotic machinery associated with CIN (see also next section).

The CEP17 probe is used in the evaluation of patients with HER2 intermediate scores in immunohistochemistry (IHC 2+) who require ISH evaluation in order to resolve HER2 status for therapeutic decisions in the clinic [16, 17]. According to established guidelines, breast cancers with a score of 2+ in IHC undergo dual probe ISH (probes for HER2 and CEP17) and are considered positive if either the ratio of HER 2 to CEP17 is above 2 or if there is an average of more than 6 signals of HER2 per tumor cell nuclei independently of the number of CEP17 signals [12]. Although HER2 positive tumors as defined by these criteria form a well-characterized subset of breast cancers with treatment implications (addition of HER2-directed therapies in treatment regimens), they have significant underlying genetic heterogeneity [18]. In addition, the repercussions of gains of CEP17 (with or without HER2 abnormalities) in breast cancer are less clear.

Several studies have attempted to characterize cancers with the amplification of CEP17 and to derive prognostic information from this molecular abnormality. Amplification of chromosome 17 centromere is present in about $10 \%$ to $40 \%$ of cases and appears to be associated with intermediate prognosis between HER2 negative and HER2 positive tumors [19-21]. Its prevalence is higher in breast cancers with amplification of the ERBB2 gene at locus $17 \mathrm{q} 12$ encoding for HER2 than in ERBB2 non-amplified tumors in some studies but shows no correlation with amplification of the ERBB2 locus in several others. A selection of studies of CEP17 amplification in breast cancer is discussed below and presented in Table 1.

Compared with HER2-negative breast cancers, breast cancers with CEP17 gain (in the study of Ji et al. defined as higher than 3.76 CEP17 copies per nucleus and not fulfilling the ISH criteria for HER2 positivity [20]) were significantly more often grade $3(12.5 \%$ versus $8 \%, \mathrm{p}=0.04)$, were more often $2+$ or $3+$ by HER 2 IHC ( $80.4 \%$ and $8.7 \%$ versus $57.2 \%$ and $2.8 \%$ respectively, $\mathrm{p}=0.000$ ) and had more often a Ki67 positivity status above $20 \%(47.3 \%$ versus $36.8 \%, \mathrm{p}=0.03)$ [20]. In addition, Disease-Free Survival (DFS) of patients with CEP17 gains was intermediate between patients with

Table 1. Examples of studies of CEP17 amplification in breast cancer. DFS: Disease Free Survival, LVI: Lymphovascular Infiltration, OS: Overall Survival, PFS: Progression Free Survival, NR: Not Reported. Cut-off refers to average copy numbers per nucleus.

\begin{tabular}{lcccll}
\hline Reference & $\begin{array}{c}\text { Number of } \\
\text { patients }\end{array}$ & Cut-off & Prevalence (\%) & Associations & Prognosis \\
\hline Ji et al. [20] & 770 & 3.76 & 23.9 & Ki67, grade & PFS intermediate \\
Lee et al. [21] & 945 & 3.0 & 19.7 & Ki67, grade, LVI & DFS worse in ER+/HER2- \\
Petroni et al. [22] & 647 & 3.7 & 27.3 & Ki67 & NR \\
Jiang et al. [23] & 109 & 2.6 & 33.9 & ERBB2 amplification, grade & NR \\
Liu et al. [24] & 348 & 3.0 & 13.8 & ER & NR \\
Foutzilas et al. [25] & 1031 & 3 & 39.9 & ERBB2, TOP2A & No OS difference \\
Gogas et al. [26] & 229 & 3.22 & 10.9 & ERBB2 amplification & No association with relapse \\
Salido et al. [27] & 175 & 3.0 & 12.5 & Nodal status & \\
\hline
\end{tabular}


HER2 negative tumors who had the best DFS and those with HER2 positive tumors who had the worse DFS [20]. Another group found that CEP17 gain (defined as a copy number of $\geq 3.0$ in this study) was present in $19.7 \%$ of breast cancers in their series of 945 patients [21]. CEP17 gain was associated with a worse DFS in the HER2 negative group but not in the HER2 positive group. No differences in DFS in either groups were noticed when a different CEP17 gain cut-off of 2.6 was used [21]. The DFS difference with the CEP17 cut-off of 3 was due to differences in the ER positive subgroup, while triple negative patients had no different DFS if they had concomitant CEP17 gain compared with no CEP17 gain. However, the number of ER and HER2 negative patients included in the series was small.

Another series showed CEP17 gain (defined as a copy number of $\geq 3.7$ ) in $27.3 \%$ (177 of 647 patients) of breast cancer patients [22]. This study found an association of cases with CEP17 gain with higher proliferation index as measured with Ki67 compared with cases without CEP17 gain. No associations with age, tumor size, nodal status or grade were discerned. Similarly, no statistically significant associations of ER status or ERBB2 amplification between cases with CEP17 gain compared with those without CEP17 gain were observed [22].

CEP17 amplification was defined as equal or more than 2.6 signals per cell in a study of 109 breast cancer patients and was associated more often with ERBB2 amplification (60\% versus $25 \%$ in cases with no CEP17 amplification) and high grade nuclear atypia than CEP17 non-amplified cases [23]. An association of CEP17 amplification with 3+ HER2 staining in immunohistochemistry was observed in this group [23].

Another series of 348 breast cancer patients from China showed a prevalence of CEP17 amplification ( $>3$ signals/ nucleus) of $13.8 \%$ [24]. There was a poor correlation of CEP17 amplification with ERBB2 amplification. Among patients with 6 or more ERBB2 signals per nucleus, $33.7 \%$ had also CEP17 amplification ( $>3$ signals/nucleus), while these percentages were $87.5 \%$ and $4 \%$ for the groups with 4 to 6 and less than 4 ERBB2 signals/nucleus, respectively [24]. Cases with CEP17 amplifications were more commonly ER positive (66.2\%) than HER2 positive patients (defined by an ERBB2/CEP17 ratio $>2.2$ ) who were ER positive in $42.2 \%$ of cases.

In a report of over a thousand breast cancer patients across sub-types that had participated in two adjuvant anthracycline trials of the Hellenic Cooperative Oncology Group (HeCOG), $39.9 \%$ of patients presented gain in CEP17, defined as more than 3 signals per nucleus in at least $30 \%$ of counted nuclei [25]. CEP17 gains had a higher prevalence in cases with increased numbers of ERBB2 signals and cases with increased numbers of TOP2A signals. Interestingly, cases with TOP2A deletions had also an increased occurrence of CEP17 gains compared with TOP2A non-amplified cases. Investigators reported also on CEP17 gain prevalence according to biological subtype. Luminal A cancers (ER-positive and/ or PR-positive, HER2-negative and Ki-67 <14\%) had
CEP17 gain in $38.4 \%$ of cases. Similarly, luminal B cancers (ER-positive and/or PR-positive, HER2-negative and Ki-67 $>14 \%$ ) had CEP17 gain in $39.4 \%$ of cases. In HER2-amplified tumors gains of CEP17 were observed in $49.3 \%$ ER-positive and $53.7 \%$ of ER-negative patients. Triple negative sub-type had a CEP17 gain in $26.2 \%$ of cases [25]. No differences in survival were observed according to CEP17 status.

A series of breast cancer patients that had been treated in HER2-positive trials of HeCOG had been investigated for copy number alterations of c-MYC, c-MET, ERBB2, TOP2 and the centromeres of the corresponding chromosomes, CEP8, CEP7 and CEP17 [26]. Some of these patients had actually been determined to be HER2 negative by central review. TOP2A amplifications and c-MYC amplifications were observed more commonly in centrally HER2-positive tumors $(46.4 \%$ and $21.8 \%$ of cases) than in centrally HER2-negative cases (6.8\% and $11.1 \%$ of cases respectively). In addition, HER2 amplified tumors by central review had more commonly amplifications of CEP17 (13.8\% versus $6.8 \%$ ) than tumors that were centrally HER2 non-amplified. In contrast no differences in amplification frequencies of CEP7 or CEP8 were observed. CEP8 was amplified in about one fourth of both HER2 positive and negative cases [26]. Thus, it appears that a significant percentage of breast cancers bear abnormalities in centromeric chromosomal areas independently of their ERBB2 amplification status.

In a study of CEP 17 "polysomy", defined as 3 or more copies per nucleus, in 175 breast cancer patients, the incidence of CEP17 gain was $12.5 \%$. No association of CEP17 gain with ERBB2 amplification has been observed [27]. CEP17 amplification was present in 15\% ERBB2 non-amplified cases and in $10 \%$ of ERBB2 amplified cases. No associations were also observed between CEP17 gain and either tumor grade or ER status [27].

Data discussed here show a high prevalence of CEP17 amplification in breast cancer and argue for a possible prognostic role of CEP17 amplification in at least sub-sets of breast cancer. If the underlying pathophysiology of CEP17 abnormalities is related to a more generalized CIN in some cases, as it will be discussed next, it could be explored for obtaining therapeutic gains. However, the fact that studies that examined other centromeric amplifications found them to be present in breast cancer commonly in the absence of CEP17 amplification may suggest that use of a combination of centromeric and arguably non-centromeric probes may be preferable for this purpose. In this line of argument clinical cases with both ERBB2 and CEP17 amplifications may have a higher CIN level than cases with only one of them or none of the two probes amplified.

Interestingly from the limited and somewhat heterogeneous data available, no clear association of ER status of the tumor with CEP17 gains is observed. Studies have shown either no statistically significant association or association of higher rates of CEP17 gains with ER positive cancers [24, 27] or conversely a higher rate of CEP17 gain in HER2 positive 
cancers than in the luminal types [25]. These data suggest that either there is no strong association of CEP17 gains with breast cancer sub-types or the heterogeneity of the studies both regarding populations included and techniques used prevent identification of sub-types differences in this regard. In addition, CEP17 gain is observed in cancers across the spectrum of HER 2 expression by IHC and thus it seems that it does not significantly influence such expression, despite the proximity of the two loci [28]. In the study of DownsKelly et al. $69 \%$ of patients with CEP17 gain were negative for HER2 ( 0 or $1+$ by IHC), $27 \%$ of patients had an intermediate score of $2+$ and only $3 \%$ of patients had an HER 2 expression of $3+$ [28]. Clearly further studies are required to confirm CEP17 role in breast cancer.

\section{Centromeres integrity and CIN in cancer}

The repetitive nature of centromeric DNA presents challenges in the maintenance of integrity and length of centromeres during their duplication before distribution to daughter cells [29]. Thus centromeric and pericentromeric regions are common sites of breaks and re-arrangements. Cancer-associated re-arranged chromosome fragments tend to be better preserved in subsequent cell generations if they are connected to a functional centromere, while they are lost in subsequent mitoses and cell divisions if they are acentric [30]. Given that centromere assembly and functionality depends on epigenetic factors, such as histone 3 variant CENP-A occupancy rather than the underlying centromere specific a satellite sequences, CENP-A overexpression protects against DNA damage induced for example by UV light [31]. In addition, DNA double breaks produce accumulation of centromeric proteins, such as CENP-A and its chaperone HJURP (Holliday Junction Recognition Protein) in broken sites $[32,33]$. This accumulation leads to the recruitment of additional repair proteins, such as hMSH5 and NBS, possibly connecting centromere production with DNA repair. Centromere formation in sites of DNA double strand breaks may constitute a back-up mechanism for rescuing DNA sequences if repair fails. Consistent with these considerations, mislocalization of CENP-A promotes CIN [34]. As a result, CEP17 amplification observed in some breast cancers as well as amplifications of other centromeres present concomitantly in the same cases or instead in other cases may become a feed forward mechanism of CIN stabilization by contributing to preservation of rearranged DNA fragments. However, CEP17 amplification as observed in breast cancer in the clinics requires the presence of amplified underlying $a$ satellite sequences of the centromere 17 , which are detected by the used ISH probe, while even functional centromeres able to assemble kinetochores would not be detected by the probe if not associated with the respective a satellites. Thus, in clinical cases with centromere amplifications the underlying a satellite sequences are amplified. Whether these amplified $\alpha$ satellites are able and do in fact assemble functional kinetochores and constitute the basis for functional centromeres remains to be clarified. Such functionality would favor survival of cancer cells with high CIN as it would protect DNA fragments from loss in daughter cells that could lead to decreased or loss of viability. For this reason, centromere amplifications may constitute an advantage for CIN-high cancers and be maintained through Darwinian selection in these cancers. However, the offered advantage may be centromere- and case-specific, depending on the sequences rescued (Figure 1A). Theoretically, if a new amplified centromere rescues a fragment bearing an important oncogene, it may provide significant advantage for the cancer cell survival and proliferation. In contrast, if the rescue operation of another amplified centromere happens to involve a fragment bearing a tumor suppressor or several tumor suppressors, it may have the opposite effect and be deleterious for the survival of the specific cancer cell. This might be the mechanism underlying the phenomenon of specific chromosomes centromere amplifications observed in some cancers [29].

\section{Chromosome 17 centromere amplification: Relationships to CIN mechanisms}

Evidence supports the association of amplifications in chromosome 17 centromere region and in the ERBB2 locus with generalized CIN, although given the higher CIN prevalence than the prevalence of CEP17 and ERBB2 gains in breast cancer, several cases without these gains may still have high levels of CIN. First, suggestive of CEP17 and ERBB2 association with CIN, there is an association of the amplification of the two regions as well as the locus of topoisomerase II gene, TOP2A, which is located telomeric to ERBB2 in arm 17q [35]. When CEP17 was amplified, ERBB2 was concomitantly amplified in $38.3 \%$ of cases, and TOP2A was amplified or deleted also in $38.3 \%$ of cases, while when CEP17 was not amplified, HER 2 was amplified in only $15 \%$ of cases and TOP2A was amplified or deleted in $10 \%$ of cases [36]. In addition, in a study that used a panel of microsatellite markers for 26 chromosomal regions, ERBB2 amplified tumors had higher genomic instability (27\% versus $19 \%$ ) than non-amplified tumors [37]. In another study examining chromosome 17 alterations in breast cancer cell lines, HER2 positive cell lines had a higher frequency of chromosome 17 rearrangements [38]. ERBB2 amplifications have also been associated with the phenomenon of chromothripsis, which consists of catastrophic rearrangements in defined genetic areas, while the rest of the genome remains intact [39].

A second line of evidence for the association of centromere lesions with CIN comes from studies in model organisms, which have shown that re-replication errors of centromere regions that lead to replication of these regions more than once in a single cell cycle, induce CIN [40]. Additionally, transfection of lentiviral vectors expressing centromeric a satellite sequences in human mammary epithelial cells promoted segregation errors and copy number altera- 

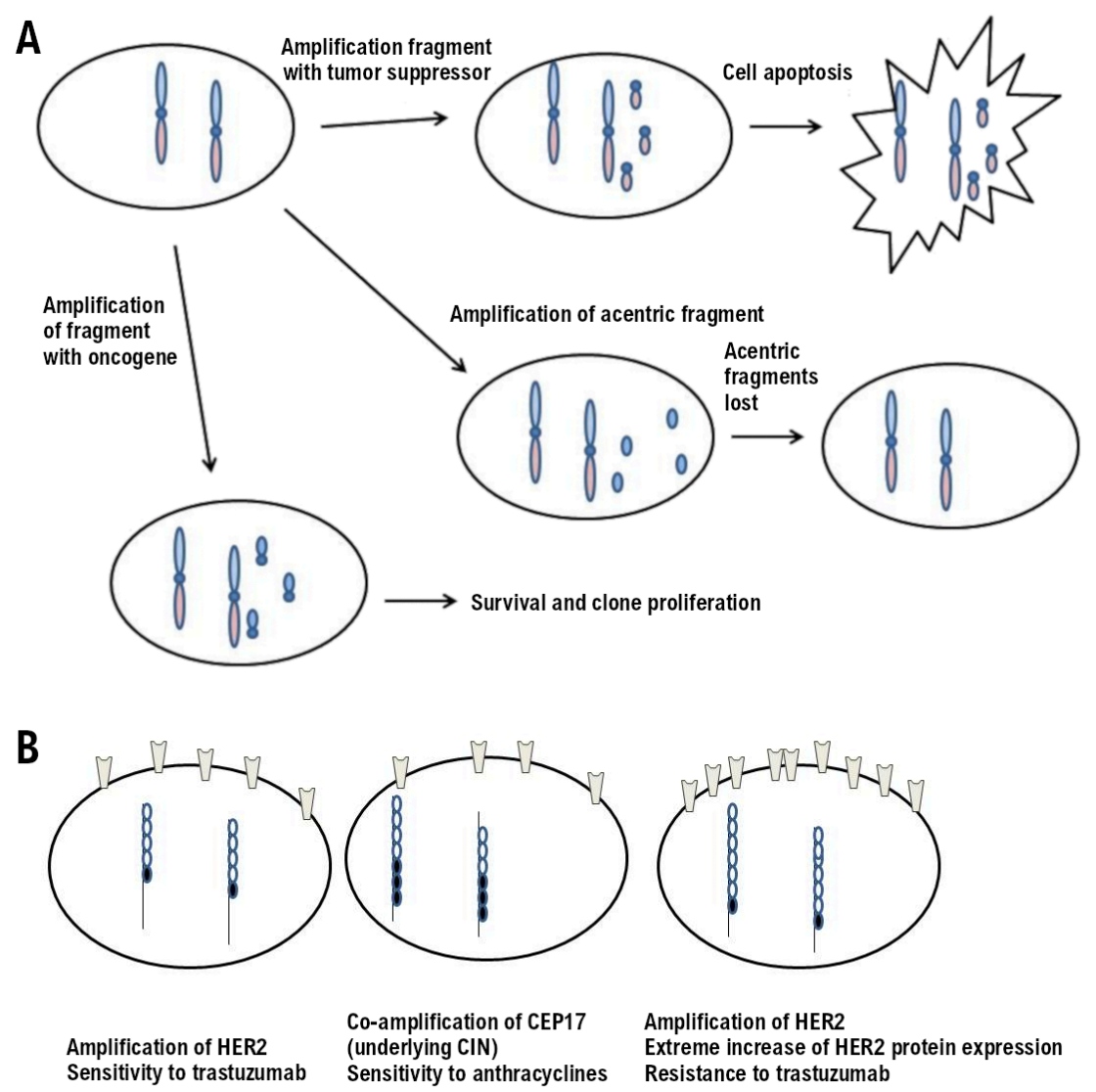

Figure 1. A) Schematic representation of possible effect of amplifications in the fate of a cancer cell. Amplification of genetic material containing a tumor suppressor (light red) would promote cell apoptosis (upper part). Amplification of material without associated functional centromere has a higher probability of loss of the amplified sequences if they are not re-incorporated in a chromosome (middle cell fate). Amplification of chromosomal parts containing oncogenes (light blue) would promote cell survival and proliferation and could be clonally selected. B) Effect of HER2 amplification in the sensitivity to treatments. Cancers with HER2 amplification without co-amplification of CEP17, implying lower CIN levels (left), are more sensitive to anti-HER2 based neo-adjuvant treatments. Co-amplification of CEP17, implying a higher level of CIN (middle), may underline increased sensitivity to anthracyclines. High levels of surface expression of the HER2 protein often produced by high levels of HER2 amplification (right) may lead to resistance to anti-HER2 treatments. Open ovals on chromosome 17 (represented by a vertical line) represent the HER2 locus and dark ovals represent the centromeric locus.

tions [41]. Overexpression of a satellites was associated with hypomethylation of these sequences. Moreover, loss of trimethylation at lysine 9 of histone 3 (H3K9me3) in pericentromeric chromatin was associated with a reduction of ability to assemble the kinetochore [42]. This would suggest a role of transcriptional silencing of the pericentromeric heterochromatin for the correct function of centromeres. Overexpression of demethylase JMJD2B that catalyzes H3K9 demethylation is associated with CIN in breast cancer and forced expression has the same effect in cell lines [42]. In contrast to the pericentromeric heterochromatic areas, centromeres bear post-translational modifications of potentially transcriptionally active chromatin, such as $\mathrm{H} 3 \mathrm{~K} 4$ dimethylation and lack transcription repressive $\mathrm{H} 3 \mathrm{~K} 9$ dimethylation [43]. Transcription of non-coding satellite centromeric areas play a role in assembling the functional centromere by producing long RNAs, which bind histone variant CENP-A carried by chaperone HJURP and contribute to the correct localization of CENP-A in the centromere [44]. The length of these RNAs that may be over a kilobase long appears to be critical, as shorter transcripts accumulate under stress or upon differentiation conditions and lead to centromere dysfunction, possibly due to CENP-A mislocalization and to chromosome missegregation [45]. Overexpression of a satellite sequences has been reported in diverse murine and human cancer cell lines as well as cancer tissues compared to matched normal tissues [46].

A third, more circumferential line of evidence for an association of amplifications of ERBB2 and CEP17 loci with generalized CIN in breast cancers is provided by treatment efficacy data in the neo-adjuvant setting (Figure 1B). In a study from the Austrian Breast and Colorectal Cancer Study Group patients with an ERBB2 to CEP17 ratio higher than 6, implying lower numbers of CEP17 copies (as these are in the denominator) and thus possibly lower degree of generalized $\mathrm{CIN}$, had a higher complete pathologic response rates (pCR) 
to neo-adjuvant trastuzumab-based treatment than patients with ERBB2 to CEP17 ratios lower than 6 (pCR of $69 \%$ versus $30.4 \%$ in patients with ERBB2/CEP17 <6) [47]. Interestingly, ER positive tumors were the drivers of the ERBB2 to CEP17 ratio association with $\mathrm{pCR}$ while ER negative tumors had no statistically different $\mathrm{pCR}$ rates whether their ERBB2 to CEP17 was higher or lower than 6. In another retrospective study of patients receiving neo-adjuvant treatment for locally advanced HER2 positive breast cancers, patients with a higher ratio of ERBB2 to CEP17, as a continuous variable but not with a cut-off of 7, had a higher rate of $\mathrm{pCR}$ than patients with lower ERBB2 to CEP17 ratios and it was independently associated with pCR in a logistic regression model [48]. In this series only 373 of the 555 patients had received trastuzumab as part of their neo-adjuvant treatment. In another study of 140 patients that received anthracycline-based neo-adjuvant chemotherapy, patients with amplified CEP17, defined as a mean of more than 1.86 signals per nucleus, were associated with higher pCR to neo-adjuvant anthracyclines [49]. With the definition used in this study, 9.5\% of the patients had CEP17 amplification. In addition, patients with amplification of topoisomerase IIa (TOP2A) showed a trend for higher pCR, while patients with ERBB2 amplification did not show different pCR compared to ERBB2 non-amplified cases. Given that anthracyclines have been proposed to be more effective in tumors with higher CIN $[50,51]$, these data may imply that CEP17 amplification is associated with CIN, and as a result, with anthracyclines sensitivity. Additional evidence from a pooled analysis of data from five adjuvant breast cancer clinical trials has indeed confirmed the predictive value of duplication of CEP17 and TOP2A aberrations, which may be surrogates for generalized CIN, for benefit from treatment with anthracyclines [52]. In contrast to the above data suggesting better clinical responses with higher ERBB2/ CEP17 copy number ratios, a study that used a method of quantification of total HER2 protein content in breast cancers from the FinHer trial found that HER2 positive cancers by
ISH with the highest HER2 protein content showed resistance to trastuzumab [53]. This result suggests that caution is needed when evaluating associations in retrospective studies, especially in trials that have used combination treatments because a specific characteristic may promote response to one component of the combination while promoting resistance to another component. In treatments of HER2 positive breast cancers employing combinations of regimens containing anthracyclines and trastuzumab (or other anti-HER2 drugs), tumors with the higher ERBB2 amplification may be particularly sensitive to anthracyclines if significant CIN is present but resistant to trastuzumab if very high protein levels of HER2 receptor that are difficult to block are also present.

In summary, CEP17 amplification in the clinical context of breast cancer HER2 evaluation may give additional information regarding CIN and if present in the same tumors with ERBB2 amplification may suggest a more generalized pathophysiologic defect that leads to CIN in the specific breast cancer. HER2 evaluation alone may not inform on CIN globally as isolated amplifications in cancer promoting loci could be a chance event stabilized through selection of the amplification, which favors cancer cells survival and propagation given that it contains the ERBB2 oncogene. CEP17 amplified areas (and amplified centromeric areas from other chromosomes) could form functional kinetochores if associated with centromere specific histone CENP-A, which is often overexpressed in breast cancer as discussed in the next section.

\section{Implications of pericentromeric chromosome 17 region abnormalities in breast cancer as derived from publicly available genomic data}

Chromosomal centromeric loci are not fit for study by currently available genomic surveys because these studies rely on an assembly of fragments of variable sizes and sequences in order to call copy number alterations. The repetitive nature

Table 2. Pericentromeric genes in the long and short arms of chromosome 17. Centromere and pericentromeric heterochromatin of chromosome 17 span between about 22M and 27M. compl.: complementary chain.

\begin{tabular}{|c|c|c|c|}
\hline Gene name & Description & Span & Gene ID number \\
\hline \multicolumn{4}{|l|}{$\mathrm{p}$ arm } \\
\hline USP22 & Ubiquitin Specific Peptidase 22 & 20999593-21043409 (compl.) & 23326 \\
\hline TMEM11 & Transmembrane protein 11 & $21197950-21214595$ & 8834 \\
\hline MAP2K3 & Mitogen activated protein kinase kinase 3 & $21284656-21315240$ & 5606 \\
\hline KCNJ12 & $\mathrm{K}^{+}$voltage-gated channel subfamily J member 12 & $21376387-21419870$ & 3768 \\
\hline \multicolumn{4}{|l|}{$\mathrm{q}$ arm } \\
\hline MIR4522 & Micro-RNA 4522 & 27293910-27293996 (compl.) & 100616277 \\
\hline WSB1 & WD repeat and SOCS box-containing 1 & $27294021-27313631$ & 26118 \\
\hline KSR1 & Kinase Suppressor of Ras 1 & $27456470-27626438$ & 8844 \\
\hline LGALS9 & Galectin 9 & $27631148-27649560$ & 3965 \\
\hline NOS2 & Nitric oxide synthase 2 & 27756766-27800529 (compl.) & 4843 \\
\hline LYRM9 & LYR motif containing 9 & $27878314-27894746$ & 201229 \\
\hline NLK & Nemo-like kinase & $28042647-28205140$ & 51701 \\
\hline
\end{tabular}


of centromeric DNA precludes such an assembly in a satellite and pericentromeric regions. The status of centromere 17 (or any other centromere) copy numbers cannot thus be directly calculated in genomic studies. An indirect inference of the copy number status of centromeric areas could be attempted by studying the copy number status of genes located very closely to the centromeric and pericentromeric areas in both the $\mathrm{q}$ and the $\mathrm{p}$ arms of the chromosome. Copy number alterations of pericentromeric genes in both $q$ and $p$ arms in the same sample could suggest involvement of the centromere per se, in these samples, and thus serve as a surrogate marker of centromere sequences amplifications that are not directly testable with the current sequencing techniques. As an exercise to examine the feasibility of this approach and derive the amplification status of chromosome 17 centromeric loci, genes located closest to centromere in the 17q1.1 locus and $17 \mathrm{p} 1.1$ locus were identified (Table 2). Chromosome 17 is well suited to serve as the subject chromosome of this exercise in breast cancer given that its centromere has been examined in multiple studies with ISH techniques and is known to be amplified in $20 \%$ to $45 \%$ of breast cancer patients as detailed in a previous section. Some of the closest genes to centromeres in 17q1.1 listed after the pericentromeric gene desert in ensemble database include miR4522, WSB1, KSR1, LGALS9, NOS2, LYRM9 and NLK (Table 2). Similarly, genes located in the area neighboring the pericentromeric heterochromatin of the short arm of chromosome 17 include USP22, TMEM11, MAP2K3 and KCNJ12 (Table 2). However, and despite the comparative high frequency of centromere 17 amplifications, as determined by ISH, data from the METABRIC and TCGA Breast cancer studies examined in the cBioPortal platform [54-57] disclosed low frequencies of abnormalities in these pericentromeric genes and notably even lower frequencies of concomitant abnormalities in both genes located in $\mathrm{p}$ and $\mathrm{q}$ arms. In the METABRIC study that included over 1900 breast cancer patients, each of these genes was amplified in $2 \%$ to $4 \%$ of cases and all of them were concomitantly amplified in just six cases, of which only one was HER2-positive [57]. Similarly, the TCGA PanCancer Atlas Invasive Breast Cancer study that included about a thousand patients disclosed CNAs in each of these genes in $2 \%$ to $5 \%$ of cases but only four cases had amplifications of all eleven genes in the short and long arm across the centromere 17 [56]. This suggests that amplifications of genetic material in chromosome 17 rarely extend across the centromere to include both short and long arms or the whole chromosome but constitute rather a quite localized phenomenon. These data agree with the aforementioned contention that CEP17 copy number gain in breast cancer is rarely due to chromosome 17 polysomy [10].

An additional approach for derivation of information on centromere status consists of studying genes known to be involved in correct centromere assembly, such as the specific centromere variant histone CENP-A, its chaperone HJURP, or protein CENP-B and CENP-C. Mutations or abnormal expression of each of these genes, as well as others involved in the same centromere assembly process, may be responsible for a sub-set of the observed cases with centromere copy number alterations and may contribute to stability of such centromere abnormalities by conferring functionality in the underlying repetitive DNA amplification. Given the importance of centromeres for prevention of aneuploidy, lesions in genes involved in correct centromere assembly would be expected to be associated with high CIN. Interestingly interrogation of the genomic TCGA PanCancer Atlas Invasive Breast Cancer study data on the cBioPortal platform disclosed that CENP-A lesions (mainly mRNA over-expression) observed in about $10.9 \%$ of cases segregate almost exclusively in the basal sub-type. Among the 112 patients with CENP-A over-expression, $92(82.1 \%)$ belonged to the basal sub-type, nine patients (8.1\%) had the luminal B sub-type, 11 patients $(9.8 \%)$ were HER2 positive and no patients with CENP-A over-expression had a luminal A disease (Figure 2A). Conversely, among basal cancers in TCGA PanCancer study, 53.8\% had CENP-A overexpression, while this percentage was $14.1 \%, 4.6 \%$ and 0 for HER2 positive, luminal B and luminal A cancers, respectively (Figure 2B). In the same study, the mean aneuploidy score for cases with a CENP-A over-expression was 15.67 (SD: 6.74) and with no CENP-A over-expression was 11.69 (SD: 7.83)
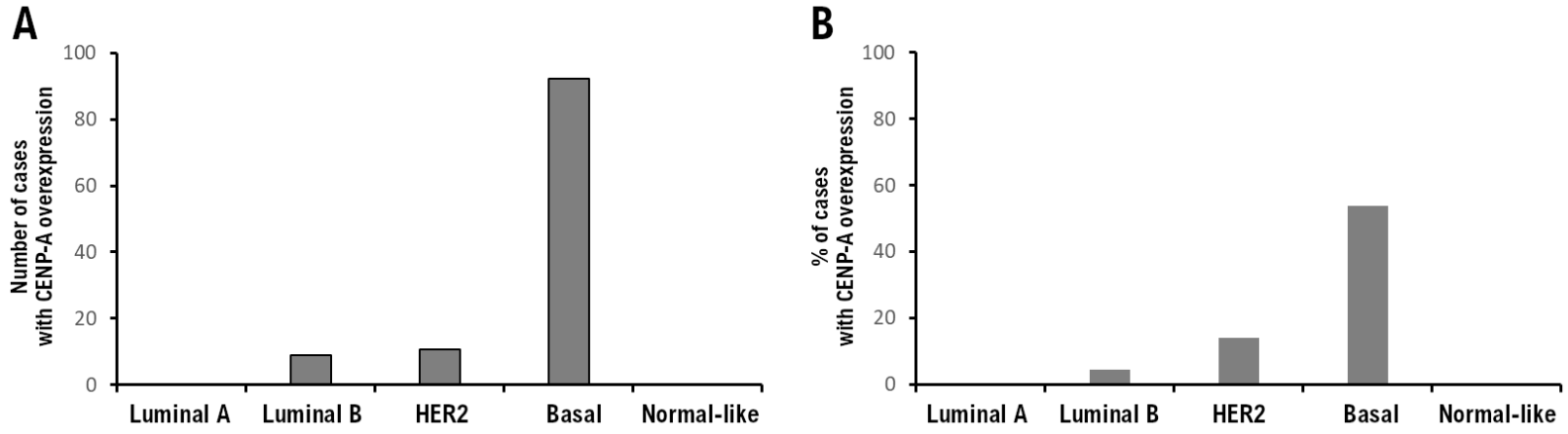

Figure 2. A) Absolute number and B) percentage of cases with breast cancer and CENP-A over-expression according to sub-type in the TCGA breast cancer PanCancer study. 
(t-test $\mathrm{p}<0.001)$. CENP-A over-expression and mislocalization has been previously reported to be associated with CIN in cancer [34]. CENP-A chaperone HJURP mRNA overexpression displays also predominance in basal cancers. HJURP was over-expressed in 69 samples in TCGA PanCancer Atlas Invasive Breast Cancer among which 55 samples (79.7\%) were basal, 10 (14.5\%) were of the luminal B sub-type, 3 samples (4.3\%) were HER2 positive, one (1.4\%) normal-like and none of the luminal A sub-type. In contrast no differences in the aneuploidy score was observed between luminal B, HER2 positive and basal cancers, despite their significant differences in the frequency of CENP-A overexpression. Aneuploidy score depicts the number of each chromosome arm with significant copy number alteration [58]. Thus, the equal aneuploidy scores of HER2 positive and luminal B cancers with this of basal cancers despite their lower frequency of CENP-A and HJURP overexpression suggests that other abnormalities play a role in these sub-types or indeed that CENP-A and HJURP overexpression is an epiphenomenon downstream of other abnormalities leading to CIN. p53 suppresses expression of CENP-A and HJURP through CDE/CHR motifs in their promoters and this may contribute to its function as a CIN suppressor [59, 60]. Suppressive action of p53 is indirect in these promoters through upregulation of $\mathrm{p} 21$, which promotes repressive p107 and p130-containing DREAM complex recruitment [61]. As a result, mutations in p53 would lead to de-repression of the two genes. This de-repression is critical to prevent excessive CIN and cell death in cancers with p53 functional loss [59]. Interrogation of data from the TCGA PanCancer Atlas Invasive Breast Cancer at cBioPortal shows a high correlation of CENP-A mRNA upregulation with mutations in p53. Among the 112 samples with upregulated CENP-A mRNA only 11 had no concomitant p53 mutation. Moreover, consistent with the upregulation of CENP-A mRNA and the role of p53 mutations in this upregulation, p53 mutations are observed in $88 \%$ of basal breast cancers but only in $17 \%$ of luminal A cancers [62].

In contrast to the DREAM complex that binds $\mathrm{CDE} / \mathrm{CHR}$ motifs and suppresses CENP-A promoter function, several other transcription regulators such as E2F1, MYBL2 (B-MYB) and FOXM may activate transcription through binding to the same motifs. All these three activating regulators have been found to be overexpressed in aneuploid breast cancers [63]. In addition, their overexpression in experimental models induces aneuploidy. The E2F family of transcription factors has members that activate and others that suppress their target promoters through binding to identical sequences and recruiting co-activators or co-repressor complexes [64]. Interestingly, a sub-set of patients from the TCGA PanCancer Atlas Invasive Breast Cancer at cBioPortal ranging from 5\% to $28 \%$ have abnormalities (mostly mRNA upregulation) in one or more members of the E2F1 family including the inhibitory members. These abnormalities are positively correlated with both CENP-A and HJURP mRNA upregulation and p53 mutations (Benjamini-Hochberg's $\mathrm{q}<0.001$ ). Thus, these data suggest an effect of these transcription factors dysregulation in overexpression of centromeric histone CENP-A and its chaperone HJURP.

It is noteworthy that despite the robust association of these transcriptional regulators with centromeric protein abnormalities and the basal phenotype, CEP17 amplifications do not display any strong prevalence association with triple negative cancers [25]. In contrast, studies that measured instability in breast cancer samples have confirmed its association with basal or HER2-positive phenotypes [4, 65]. Thus, CEP17 amplification measurement alone may not be optimally depicting the CIN status of a tumor and a more global measurement is required.

Besides CENP-A and HJURP, several other proteins (MAD1L1, MAD2L1, MAD2L2, BUB1, BUB1B, BUB3, CDC20 and TTK) that associate with the kinetochore during mitosis and participate in mitotic checkpoint are overexpressed both at the mRNA and protein level in breast cancer cell lines with genomic instability and patient samples checked [66]. An association of increased expression of BUB1B protein with grade 3 cancers was also observed in this study. MAD2L1 overexpression, in addition to CENP-A overexpression, was critical in CIN development in cells with pRB loss which upregulates E2F1 [67, 68]. In TCGA PanCancer Atlas Invasive Breast Cancer samples these genes show abnormalities (mostly increased expression) in 5\% to $12 \%$ of cases each. Basal cancers are over-represented in cases with overexpression of these mitotic regulators (data not shown).

\section{Conclusions and perspectives}

CIN, either numeric or structural and most often both, is very commonly present in cancer cells and the great majority of cancers display some degree of CIN. Its measurement in clinical samples has proven to be difficult to realize in a manner that will be clinically exploitable for diagnostic, prognostic and therapeutic purposes. CIN is a continuum of states and scales to assess and its quantification will need to be constructed and then validated before entered in standard clinical practice. Such measurements could be direct, consisting of evaluation of chromosome arm with copy number abnormalities akin to the aneuploidy score that currently is used only as a research tool [58] or indirect, consisting of measurement of genes associated with CIN, such as the CIN4 score proposed by Szász et al. [69]. The four genes included in this signature, AURKA, FOXM, TOP2A and TPX2 code all for important proteins in the mitotic spindle function and regulation.

Data discussed in this paper argue for the association of centromeric abnormalities and CIN and put forward the idea of centromere 17 amplification, possibly examined in association with other amplifications such as those of chromosome 2 and 15 [70], as a measure for CIN in breast cancer. CIN measurement could assist in breast cancer prognostication and therapy prediction, supplementing other methods. 
The advantage of a well-defined quantitative measurement together with the fact that ISH-based methods are already used in the clinic could be an asset in such use, although clinical validation for the new use would still be required. Selection of additional centromeric probes besides CEP17 and optimal cut-offs for prognostication would be critical, especially given that the prognostic implications of CIN may not be linear $[70,71]$. In addition, the implications of CIN might not be the same in different sub-types of breast cancer. Progress in answer these questions holds the potential of significant advancements in breast cancer prognosis and therapy.

\section{References}

[1] MCGRANAHAN N, BURRELL RA, ENDESFELDER D, NOVELLI MR, SWANTON C. Cancer chromosomal instability: therapeutic and diagnostic challenges. EMBO Rep 2012; 13: 528-538. https://doi.org/10.1038/embor.2012.61

[2] CRASTA K, GANEM NJ, DAGHER R, LANTERMANN $\mathrm{AB}$, IVANOVA EV et al. DNA breaks and chromosome pulverization from errors in mitosis. Nature 2012; 482: 53-58. https://doi.org/10.1038/nature10802

[3] ROYLANCE R, ENDESFELDER D, JAMAL-HANJANI M, BURRELL RA, GORMAN $P$ et al. Expression of regulators of mitotic fidelity are associated with intercellular heterogeneity and chromosomal instability in primary breast cancer. Breast Cancer Res Treat 2014; 148: 221-229. https://doi. org/10.1007/s10549-014-3153-x

[4] SMID M, HOES M, SIEUWERTS AM, SLEIJFER S, ZHANG $\mathrm{Y}$ et al. Patterns and incidence of chromosomal instability and their prognostic relevance in breast cancer subtypes. Breast Cancer Res Treat 2011; 128: 23-30. https://doi. org/10.1007/s10549-010-1026-5

[5] MOELANS CB, DE WEGER RA, VAN DIEST PJ. Absence of chromosome 17 polysomy in breast cancer: analysis by CEP17 chromogenic in situ hybridization and multiplex ligation-dependent probe amplification. Breast Cancer Res Treat 2010; 120: 1-7. https://doi.org/10.1007/s10549-0090539-2

[6] MOELANS CB, VAN DIEST PJ. CEP17 copy number increase does not indicate polysomy 17. J Clin Pathol 2014; 67: 454-455. https://doi.org/10.1136/jclinpath-2013-202104

[7] JACOT W, FICHE M, ZAMAN K, WOLFER A, LAMY PJ. The HER2 amplicon in breast cancer: Topoisomerase IIA and beyond. Biochim Biophys Acta 2013; 1836: 146-157. https://doi.org/10.1016/j.bbcan.2013.04.004

[8] KLEIVI SAHLBERG K, HONGISTO V, EDGREN $\mathrm{H}$, MÄKELÄ R, HELLSTRÖM K et al. The HER2 amplicon includes several genes required for the growth and survival of HER2 positive breast cancer cells. Mol Oncol 2013; 7: 392401. https://doi.org/10.1016/j.molonc.2012.10.012

[9] MOELANS CB, REIS-FILHO JS, VAN DIEST PJ. Implications of rarity of chromosome 17 polysomy in breast cancer. Lancet Oncol 2011; 12: 1087-1089. https://doi.org/10.1016/ S1470-2045(11)70234-0
[10] VIALE G. Be precise! The need to consider the mechanisms for CEP17 copy number changes in breast cancer. J Pathol 2009; 219: 1-2. https://doi.org/10.1002/path.2593

[11] MARCHIÒ C, LAMBROS MB, GUGLIOTTA P, VERDUN DI CANTOGNO L, BOTTA C et al. Does chromosome 17 centromere copy number predict polysomy in breast cancer? A fluorescence in situ hybridization and microarraybased CGH analysis. J Pathol 2009; 219: 16-24. https://doi. org/10.1002/path.2574

[12] WOLFF AC, HALE HAMMOND ME, ALLISON KH, HARVEY BE, MANGU PB et al. Human Epidermal Growth Factor Receptor 2 testing in breast cancer: American Society of Clinical Oncology/ College of American Pathologists Clinical practice guideline focused update. J Clin Oncol 2018; 36: 2105-2122. https://doi.org/10.1200/JCO.2018.77.8738

[13] ISOLA J, CHU L, DEVRIES S, MATSUMURA K, CHEW K, LJUNG BM, WALDMAN FM. Genetic alterations in ERBB2-amplified breast carcinomas. Clin Cancer Res 1999; 5: 4140-4145.

[14] SULLIVAN LL, CHEW K, SULLIVAN BA. A satellite DNA variation and function of the human centromere. Nucleus 2017; 8: 331-339. https://doi.org/10.1080/19491034.2017.13 08989

[15] MUSACCHIO A, DESAI A. A molecular view of kinetochore assembly and function. Biology (Basel) 2017; 6. https:// doi.org/10.3390/biology6010005

[16] PODOLL M. HER2 (c-erbB2) breast. PathologyOutlines. com website. http://www.pathologyoutlines.com/imgau/ breastmalignanther2Reisenbichler04.jpg. Accessed May 7th, 2019.

[17] GEIERSBACH K. Laboratory testing for Her2 status in breast cancer. Online: www.arup.utah.edu. Accessed May 7th, 2019.

[18] VOUTSADAKIS IA. HER2 in stemness and epithelial-mesenchymal plasticity of breast cancer. Clin Transl Oncol 2019; 21: 539-555. https://doi.org/10.1007/s12094-018-1961-x

[19] BARTLETT JMS, MUNRO AF, DUNN JA, MCCONKEY C, JORDAN S et al. Predictive markers of anthracycline benefit: a prospectively planned analysis of the UK National Epirubicin Adjuvant Trial (NEAT/ BR9601). Lancet Oncol 2010; 11 : 266-274. https://doi.org/10.1016/S1470-2045(10)70006-

[20] JI H, XUAN Q, NANDING A, ZHANG H, ZHANG Q. The clinicopathologic and prognostic value of altered chromosome 17 centromere copy number in HER2 Fish equivocal breast carcinomas. PLoS ONE 2015; 10: e0132824. https:// doi.org/10.1371/journal.pone.0132824

[21] LEE K, JANG MH, CHUNG YR, LEE Y, KANG et al. Prognostic significance of centromere 17 copy number gain in breast cancer depends on breast cancer subtype. Hum Pathol 2017; 61: 111-120. https://doi.org/10.1016/j.humpath.2016.12.004

[22] PETRONI S, ADDATI T, MATTIOLI E, CAPONIO MA, QUERO C et al. Centromere 17 copy number alteration: Negative prognostic factor in invasive breast cancer? Arch Pathol Lab Med 2012; 136: 993-1000. https://doi. org/10.5858/arpa.2011-0327-OA 
[23] JIANG H, BAI X, MENG F, ZHANG C, ZHANG X. Evaluation of chromosome 17 polysomy in breast cancer by FISH analysis of whole nuclei, and its clinicopathological significance. Oncol Lett 2014; 7: 1954-1958. https://doi. org/10.3892/ol.2014.2001

[24] LIU Y, MA L, LIU D, YANG Z, YANG C et al. Impact of polysomy 17 on HER2 testing of invasive breast cancer patients. Int J Clin Exp Pathol 2014; 7: 163-173.

[25] FOUNTZILAS G, DAFNI U, BOBOS M, KOTOULA V, BATISTATOU A et al. Evaluation of the prognostic role of centromere 17 gain and HER2/ topoisomerase II alpha gene status and protein expression in patients with breast cancer treated with anthracycline-containing adjuvant chemotherapy: pooled analysis of two Hellenic Cooperative Oncology Group (HeCOG) phase III trials. BMC Cancer 2013; 13: 163. https://doi.org/10.1186/1471-2407-13-163

[26] GOGAS H, COTOULA V, ALEXOPOULOU Z, CHRISTODOULOU C, KOSTOPOULOS I et al. MYC copy gain, chromosomal instability and PI3K activation as potential markers of unfavourable outcome in trastuzumab-treated patients with metastatic breast cancer. J Transl Med 2016; 14: 136. https://doi.org/10.1186/s12967-016-0883-z

[27] SALIDO M, TUSQUETS I, COROMINAS JM, SUAREZ M, ESPINET B et al. Polysomy of chromosome 17 in breast cancer tumors showing an overexpression of ERBB2: a study of 175 cases using fluorescence in situ hybridization and immunohistochemistry. Breast Cancer Res 2005; 7: R267-R273. https://doi.org/10.1186/bcr996

[28] DOWNS-KELLY E, YODER BJ, STOLER M, TUBBS RR, SKACEL $M$ et al. The influence of polysomy 17 on HER2 gene and protein expression in adenocarcinoma of the breast: a fluorescent in situ hybridization, immunohistochemical, and isotopic mRNA in situ hybriidization study. Am J Surg Pathol 2005; 29: 1221-1227. https://doi.org/10.1097/01. pas.0000165528.78945.95

[29] BARRA V, FACHINETTI D. The dark side of centromeres: types, causes and consequences of structural abnormalities implicating centromeric DNA. Nat Commun 2018; 9: 4340. https://doi.org/10.1038/s41467-018-06545-y

[30] POOT M. Neocentromeres to the rescue of acentric chromosome fragments. Mol Syndromol 2017; 8: 279-281. https:// doi.org/10.1159/000481332

[31] LACOSTE N, WOOLFE A, TACHIWANA H, GAREA AV, BARTH T et al. Mislocalization of the centromeric histone variant CenH3/ CENP-A in human cells depends on the chaperone DAXX. Mol Cell 2014; 53: 631-644. https://doi. org/10.1016/j.molcel.2014.01.018

[32] KATO T, SATO N, HAYAMA S, YAMABUKI T, ITO T et al. Activation of Holliday Junction-Recognizing Protein involved in the chromosomal stability and immortality of cancer cells. Cancer Res 2007; 67: 8544-8553. https://doi. org/10.1158/0008-5472.CAN-07-1307

[33] ZEITLIN SG, BAKER NM, CHAPADOS BR, SOUTOGLOU E, WANG JYJ et al. Double-strand DNA breaks recruit the centromeric histone CENP-A. Proc Natl Acad Sci U.S.A. 2009; 106: 15762-15767. https://doi.org/10.1073/ pnas.0908233106
[34] SHRESTHA RL, AHN GS, STAPLES MI, SATHYAN KM, KARPOVA TS et al. Mislocalization of centromeric histone H3 variant CENP-A contributes to chromosomal instability (CIN) in human cells. Oncotarget 2017; 8: 46781-46800. https://doi.org/10.18632/oncotarget.18108

[35] ARRIOLA E, MARCHIO C, TAN DSP, DRURY SC, LAMBROS MB et al. Genomic analysis of the HER2/ TOP2A amplicon in breast cancer and breast cancer cell lines. Lab Invest 2008; 88: 491-503. https://doi.org/10.1038/labinvest.2008.19

[36] KIM A, SHIN CS, BAE YK, KIM MK, KANG SH et al. Multiplication of chromosome 17 centromere is associated with prognosis in patients with invasive breast cancers exhibiting normal HER2 and TOP2A status. J Breast Cancer 2012; 15: 24-33. https://doi.org/10.4048/jbc.2012.15.1.24

[37] ELLSWORTH RE, ELLSWORTH DL, PATNEY HL, DEYARMIN B, LOVE B et al. Amplification of HER2 is a marker for global genomic instability. BMC Cancer 2008; 8: 297. https://doi.org/10.1186/1471-2407-8-297

[38] RONDÓN-LAGOS M, VERDUN DI CANTOGNO L, RANGEL N, MELE T, RAMÍREZ-CLAVIJO SR et al. Unraveling the chromosome 17 patterns of FISH in interphase nuclei: an in-depth analysis of the HER2 amplicon and chromosome 17 centromere by karyotyping, FISH and M-FISH in breast cancer cells. BMC Cancer 2014; 14: 922. https://doi. org/10.1186/1471-2407-14-922

[39] CHEN H, SINGH RR, LU X, HUO L, YAO H et al. Genomewide copy number aberrations and HER2 and FGFR1 alterations in primary breast cancer by molecular inversion probe microarray. Oncotarget 2017; 8: 10845-10857. https://doi. org/10.18632/oncotarget.14802

[40] HANLON SL, LI JJ. Re-replication of a centromere induces chromosomal instability and aneuploidy. PLoS Genet 2015; 11: e1005039. https://doi.org/10.1371/journal.pgen.1005039

[41] ICHIDA K, SUZUKI K, FUKUI T, TAKAYAMA Y, KAKIZAWA $\mathrm{N}$ et al. Overexpression of satellite alpha transcripts leads to chromosomal instability via segregation errors at specific chromosomes. Int J Oncol 2018. https://doi. org/10.3892/ijo.2018.432

[42] SLEE RB, STEINER CM, HERBERT BS, VANCE GH, HICKEY RJ et al. Cancer-associated alteration of pericentromeric heterochromatin may contribute to chromosome instability. Oncogene 2012; 31: 3244-3253. https://doi.org/10.1038/ onc.2011.502

[43] SULLIVAN BA, KARPEN GH. Centromeric chromatin exhibits a histone modification pattern that is distinct from both euchromatin and heterochromatin. Nat Struct Mol Biol 2004; 11: 1076-1083. https://doi.org/10.1038/nsmb845

[44] QUÉNET D, DALAL Y. A long non-coding RNA is required for targeting centromeric protein A to the human centromere. eLife 2014; 3: e03254. https://doi.org/10.7554/ eLife.03254

[45] BOUZINBA-SEGARD H, GUAIS A, FRANCASTEL C. Accumulation of small murine minor satellite transcripts leads to impaired centromeric architecture and function. Proc Natl Acad Sci U S A 2006; 103: 8709-8714. https://doi. org/10.1073/pnas.0508006103 
[46] TING DT, LIPSON D, PAUL S, BRANNIGAN BW, AKHAVANFARD $S$ et al. Aberrant overexpression of satellite repeats in pancreatic and other epithelial cancers. Science 2011; 331: 593-596. https://doi.org/10.1126/science.1200801

[47] SINGER CF, TAN YY, FITZAL F, STEGER GG, EGLE D et al. Pathological complete response to neoadjuvant trastuzumab is dependent on HER2/CEP17 ratio in HER2-amplified early breast cancer. Clin Cancer Res 2017; 23: 3676-3683. https:// doi.org/10.1158/1078-0432.CCR-16-2373

[48] KOGAWA T, FOUAD TM, LIU DD, WU J, SHEN Y et al. High HER2/ centromeric probe for chromosome 17 Fluorescence In Situ Hybridization ratio predicts pathologic complete response and survival outcome in patients receiving neoadjuvant systemic therapy with trastuzumab for HER2-overexpressing locally advanced breast cancer. Oncologist 2016; 21: 21-27. https://doi.org/10.1634/theoncologist.2015-0101

[49] TIBAU A, LÓPEZ-VILARÓ L, PÉREZ-OLABARRIA M, VÁZQUEZ T, PONS C et al. Chromosome 17 centromere duplication and responsiveness to anthracycline-based neoadjuvant chemotherapy in breast cancer. Neoplasia 2014; 16 : 861-867. https://doi.org/10.1016/j.neo.2014.08.012

[50] BURRELL RA, JUUL N, JOHNSTON SR, REIS-FILHO JS, SZALLASI Z, SWANTON C. Targeting chromosomal instability and tumour heterogeneity in HER2-positive breast cancer. J Cell Biochem 2010; 111: 782-790. https://doi. org/10.1002/jcb.22781

[51] SPEARS M, YOUSIF F, LYTTLE N, BOUTROS PC, MUNRO $\mathrm{AF}$ et al. A four gene signature predicts benefit from anthracyclines: evidence from the BR9601 and MA.5 clinical trials. Oncotarget 2015; 6: 31693-31701. https://doi.org/10.18632/ oncotarget.5562

[52] BARTLETT JMS, MCCONKEY CC, MUNRO AF et al. Predicting anthracycline benefit: TOP2A and CEP17-Not only but also. J Clin Oncol 2015; 33: 1680-1687. https://doi. org/10.1200/JCO.2013.54.7869

[53] JOENSUU H, SPERINDE J, LEINONEN M, HUANG W, WEIDLER J et al. Very high quantitative tumor HER2 content and outcome in early breast cancer. Ann Oncol 2007; 22: 2007-2013. https://doi.org/10.1093/annonc/mdq710

[54] CERAMI E, GAO J, DOGRUSOZ U, GROSS BE, SUMER SO et al. The cBio Cancer Genomics Portal: An open platform for exploring multidimensional cancer genomics data. Cancer Discov 2012; 2: 401-404. https://doi.org/10.1158/21598290.CD-12-0095

[55] GAO J, AKSOY BA, DOGRUSOZ U, DRESDNER G, GROSS $B$ et al. Integrative analysis of complex cancer genomics and clinical profiles using the cBioPortal. Sci Signal 2013; 6: pl1. https://doi.org/10.1126/scisignal.2004088

[56] THE CANCER GENOME ATLAS NETWORK. Comprehensive molecular portraits of human breast tumors. Nature 2012; 490: 61-70. https://doi.org/10.1038/nature11412

[57] CURTIS C, SHAH SP, CHIN S-F, TURASHVILLI G, RUE$\mathrm{DA} O \mathrm{OM}$ et al. The genomic and transcriptomic architecture of 2,000 breast tumours reveals novel subgroups. Nature 2012; 486: 346-352. https://doi.org/10.1038/nature10983

[58] TAYLOR AM, SHIH J, HA G, GAO GF, ZHANG X et al. Genomic and functional approaches to understanding cancer aneuploidy. Cancer Cell 2018; 33: 676-689. https://doi. org/10.1016/j.ccell.2018.03.007
[59] FILIPESCU D, NAUGHTIN M, PODSYPANINA K, LEJOUR V, WILSON L et al. Essential role for centromeric factors following p53 loss and oncogenic transformation. Genes Dev 2017; 31: 463-480. https://doi.org/10.1101/ gad.290924.116

[60] AYLONY,ORENM.p53: Guardian of ploidy. MolOncol2011; 5: 315-323. https://doi.org/10.1016/j.molonc.2011.07.007

[61] ENGELAND K. Cell cycle arrest through indirect transcriptional repression by p53: I have a DREAM. Cell Death Differ 2018; 25: 114-132. https://doi.org/10.1038/cdd.2017.17

[62] BERTHEAU P, LEHMANN-CHE J, VARNA M, DUMAY A, POIROT B et al. p53 in breast cancer subtypes and new insights into response to chemotherapy. Breast 2013; 22: S27S29. https://doi.org/10.1016/j.breast.2013.07.005

[63] PFISTER K, PIPKA JL, CHIANG C, LIU Y, CLARK RA et al. Identification of drivers of aneuploidy in breast tumors. Cell Rep 2018; 23: 2758-2769. https://doi.org/10.1016/j.celrep.2018.04.102

[64] MENG P, GHOSH R. Transcription addiction: can we garner the Yin and Yang functions of E2F1 for cancer therapy? Cell Death Dis 2014; 5: e1360. https://doi.org/10.1038/cddis.2014.326

[65] HABERMANN JK, DOERING J, HAUTANIEMI S, ROBLICK UJ, BÜNDGEN NK et al. The gene expression signature of genomic instability in breast cancer is an independent predictor of clinical outcome. Int J Cancer 2009; 124: 1552-1564. https://doi.org/10.1002/ijc.24017

[66] YUAN B, XU Y, WOO JH, WANG Y, BAE YK et al. Increased expression of mitotic checkpoint genes in breast cancer cells with chromosomal instability. Clin Cancer Res 2006; 12: 405-410. https://doi.org/10.1158/1078-0432.CCR-05-0903

[67] AMATO A, LENTINI L, SCHILLACI T, IOVINO F, DI LEONARDO A. RNAi mediated acute depletion of Retinoblastoma protein $(\mathrm{pRb})$ promotes aneuploidyin human primary cells via macronuclei formation. BMC Cell Biol 2009; 10: 79. https://doi.org/10.1186/1471-2121-10-79

[68] SCHVARTZMAN JM, DUIJF PHG, SOTILLO R, COKER C, BENEZRA R. Mad2 is a critical mediator of the chromosome instability observed upon $\mathrm{Rb}$ and p53 pathway inhibition. Cancer Cell 2011; 19: 701-714. https://doi.org/10.1016/j. ccr.2011.04.017

[69] SZÁSZ AM, LI Q, EKLUND AC, SZTUPINSZKI Z, ROWAN A et al. The CIN4 chromosomal instability qPCR classifier defines tumor aneuploidy and stratifies outcome in grade 2 breast cancer. PLoS ONE 2013; 8: e56707. https:// doi.org/10.1371/journal.pone.0056707

[70] ROYLANCE R, ENDESFELDER D, GORMAN P, BURRELL RA, SANDER J et al. Relationship of extreme chromosomal instability with long-term survival in a retrospective analysis of primary breast cancer. Cancer Epidemiol Biomarkers Prev 2011; 20: 2183-2194. https://doi.org/10.1158/1055-9965. EPI-11-0343

[71] BIRKBAK NJ, EKLUND AC, LI Q, MCCLELLAND SE, ENDESFELDER D et al. Paradoxical relationship between chromosomal instability and survival outcome in cancer. Cancer Res 2011; 71: 3447-3452. https://doi. org/10.1158/0008-5472.CAN-10-3667 